\title{
Correction to: Investigation of brain structure in the 1-month infant
}

\author{
Douglas C. Dean III, ${ }^{1,2}$ E. M. Planalp ${ }^{1,3} \cdot$ W. Wooten ${ }^{2} \cdot$ C. K. Schmidt ${ }^{1,2} \cdot$ S. R. Kecskemeti ${ }^{1}$. C. Frye ${ }^{2} \cdot$ N. L. Schmidt ${ }^{1,2}$. \\ H. H. Goldsmith ${ }^{1,3} \cdot$ A. L. Alexander ${ }^{1,4,5} \cdot$ R. J. Davidson ${ }^{1,2,3,4}$
}

Published online: 10 March 2018

c) Springer-Verlag GmbH Germany, part of Springer Nature 2018

\section{Correction to: Brain Structure and Function https://doi.org/10.1007/s00429-017-1600}

The authors regret that, in this article, there was an error in the analyses comparing infant male and female regional brain volume differences. In Table 3 of the original manuscript, we accurately present the mean infant male and female brain volumes. However, ANOVA analyses comparing the male and female regional brain volumes were miscalculated. Percent differences, though accurately calculated for raw regional brain volumes, were misattributed during interpretation of our findings. The first five columns of the original Table 3 correspond to analyses not controlling for total brain volume and age; the last column, 'Direction', presented findings of analyses that were calculated after controlling for total brain volume and infant age. This was not clearly described in the original manuscript. We inadvertently interpreted the findings from the corrected and uncorrected analyses together, resulting in incorrect conclusions regarding sex differences in the infant brain after controlling for total brain volume and age. Only results pertaining to the comparison of regional brain volume differences between males and females were affected.

The original article can be found online at https://doi.org/10.1007/ s00429-017-1600-2.

Douglas C. Dean III

deaniii@wisc.edu

1 Waisman Center, University of Wisconsin-Madison, Madison, WI 53705, USA

2 Center for Healthy Minds, University of Wisconsin-Madison, Madison, WI, USA

3 Department of Psychology, University of Wisconsin-Madison, Madison, WI, USA

4 Department of Psychiatry, University of Wisconsin-Madison School of Medicine and Public Health, Madison, WI, USA

5 Department of Medical Physics, University of Wisconsin-Madison School of Medicine and Public Health, Madison, WI, USA
We have reevaluated analyses examining differences between male and female regional brain volumes and clarified our results in a revised table as part of this correction. This table more clearly distinguishes analyses between male and female differences in regional brain volumes prior to and after controlling for total brain volume and age. After controlling for total brain volume and age, the male and female differences follow the same direction as originally indicated in the last column of Table 3 but are not significant.

In summary, the major ramification of the error reported here is a change in the extent to which regional brain volumes differ between males and females at 1 month of age and our conclusions regarding such findings. These amended results more clearly align with the previous neuroimaging reports of childhood and adolescent sex differences. The amended results indicate that raw sex differences in specific regions of interest are driven by total brain volume sex differences. Table 1 of this correction provides an updated version of Table 3 from the original manuscript and describes regional brain differences between males and females prior to and after controlling for total brain volume and age.

All other results of the original manuscript pertaining to age, asymmetry, and interactions among predictor variables were presented and discussed accurately.

The authors apologize for this error. 


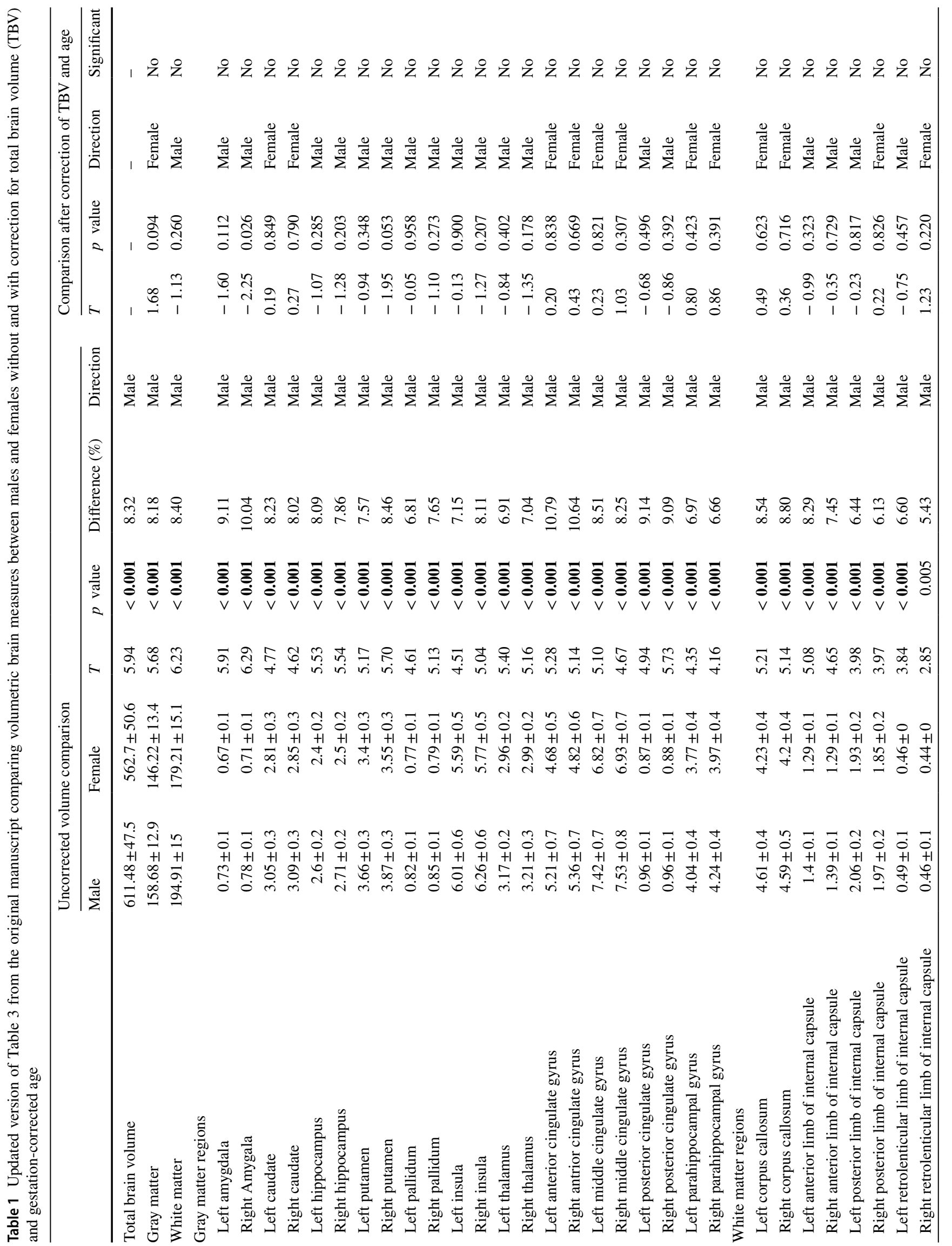




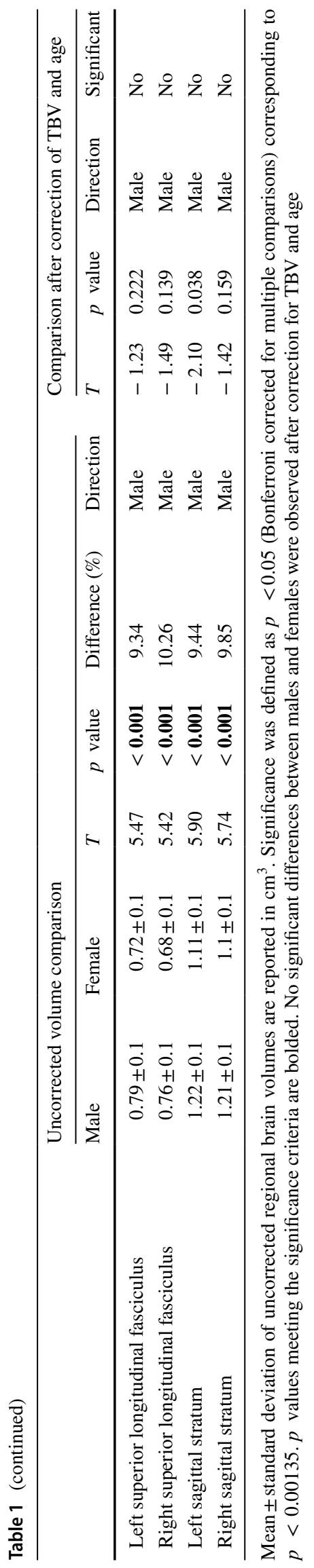

